\title{
From Prague to Seattle: Improved Endoscopic Technique and Reporting Improves Outcomes in Patients with Barrett's Esophagus
}

\author{
J. Brown ${ }^{1,2} \cdot$ P. Sharma ${ }^{1,2}$
}

Published online: 7 November 2015

(c) Springer Science+Business Media New York 2015

Endoscopic screening and surveillance practices in patients with Barrett's esophagus (BE) remain controversial. No randomized, controlled trials have supported decreased mortality as a result of these programs, but given evidence from cohort and retrospective studies combined with the rapidly rising incidence of esophageal adenocarcinoma (EAC), some GI societies have weakly recommended screening and surveillance endoscopy for BE patients [1]. The goal of the initial endoscopy is to detect BE and, if detected, to grade and evaluate for the presence of dysplasia and EAC.

In this issue of Digestive Diseases and Sciences, Visrodia et al. [2] report the proportion of cases of "missed dysplasia" diagnosed at repeat endoscopy in comparison with the index endoscopy findings. In this retrospective study, patients who underwent initial endoscopy from 1976 to 2011, with diagnosed $\mathrm{BE}$ of length $1 \mathrm{~cm}$ or greater, i.e., columnar mucosa with intestinal metaplasia, and who had undergone repeat endoscopy within 24 months of the index examination, were included in the analysis. Notably, the rate of missed high-grade dysplasia and EAC was $1.9 \%$, whereas the overall rate of missed dysplasia of any grade and/or EAC was $9.5 \%$. While the study has limitations, including its retrospective nature, questionable adherence to guidelines, adequate number of biopsies, and the types

P. Sharma

PSHARMA@kumc.edu

1 Division of Gastroenterology and Hepatology, Veterans Affairs Medical Center, University of Kansas School of Medicine, Kansas City, MO, USA

2 Division of Gastroenterology, Department of Medicine, University of Kansas School of Medicine, Kansas City, MO, USA of endoscopes used, it does highlight an important issuethe importance of meticulous endoscopy and biopsy at the time of index endoscopy in BE patients.

If during index endoscopy $\mathrm{BE}$ is confirmed or suspected, the extent of BE should be carefully documented using the Prague C\&M criteria. As part of this exercise, the landmarks that should be evaluated include the squamocolumnar junction, the gastroesophageal junction, and the extent of BE. Since this classification describes the circumferential $(\mathrm{C})$ columnar lining and the maximum length (M) of BE, excluding islands [1], in this fashion it provides a standardized description of $\mathrm{BE}$, useful for comparison with subsequent endoscopic evaluations. It also facilitates communication among gastroenterologists when patients are referred to tertiary centers for treatment. Despite the benefits of the Prague classification, a recent study reported that a surprising $78 \%$ of gastroenterologists across varying practice settings did not use the Prague classification [3].

Other considerations during index endoscopy that increase the value of the examination are careful inspection of the BE mucosa, the time spent in its evaluation, and the choice of imaging modalities. For instance, longer inspection times during endoscopic evaluation are associated with higher detection rates for HGD. A recent study reported that when endoscopists spent $\sim 1 \mathrm{~min} / \mathrm{cm}$ extent of $\mathrm{BE}$, the detection rate for neoplastic lesions improved [4]. Regarding the choice of imaging modality, high-definition imaging is superior to standard-definition imaging for BE. For example, narrowband imaging (NBI) enables evaluation of the mucosal patterns superior to that of conventional endoscopic imaging with a reported sensitivity of $100 \%$ and a specificity of $98.7 \%$ [5], due to the identification of abnormal pit and vasculature patterns within the mucosa. Thus, the combination of additional time spent assessing the mucosa along with advanced 
imaging through improved targeting of biopsies provides a greater probability of identifying BE, dysplasia, and EAC.

After careful examination of the BE mucosa, biopsies should be obtained based on the Seattle protocol, i.e., biopsies should be obtained in 4 quadrants every $2 \mathrm{~cm}$. Poor adherence to the Seattle protocol can significantly reduce the probability of successful neoplasia detection [6, 7]. Further, if there is suspicion for dysplasia, the biopsy specimens should be reviewed and evaluated by an experienced GI pathologist, since dysplasia interpretation can be problematic; suboptimal inter-observer agreement, particularly for low-grade dysplasia, is frequent with overall $\kappa$ values reported as low as 0.11 for low-grade dysplasia even among expert pathologists [8]. Increased BE extent can also contribute to missed dysplasia [9] as poorer adherence to the Seattle protocol in patients with longer segments of $\mathrm{BE}$ has been reported [10, 11].

In summary, given the potential for "missed dysplasia," the study by Visrodia and colleagues emphasizes the importance of a carefully performed index endoscopy combined with adequate and well-placed biopsies when evaluating patients with known or suspected BE. Clearly, after the initial successes reported for colonoscopic technique in the detection of colon polyps and cancer, gastroenterologists are now paying increased attention to the thoroughness of their endoscopic examination for BE. Careful endoscopic technique and an increase in the duration of the endoscopic examination of BE mucosa are first steps, along with the use of the Prague classification for descriptive standardization. Advancements in imaging currently underway holds promise for detection of abnormalities within BE for improved targeting of biopsies. The movement toward careful endoscopy in patients with BE is underway; with its success should come improved clinical outcomes.

\section{References}

1. Wang, et al. Updated guidelines 2008 for the diagnosis, surveillance and therapy of Barrett's Esophagus. Am J Gastroenterol. 2008;103:788-797.

2. Visrodia K, Iyer PG, Schleck CD, Zinsmeister AR, Katzka DA. Yield of repeat endoscopy in Barrett's esophagus with no dysplasia and low-grade dysplasia: a population-based study. Dig Dis Sci. (Epub ahead of print). doi:10.1007/s10620-015-3697-6.

3. Singh $\mathrm{M}$, et al. Practice patterns among US gastroenterologists regarding endoscopic management of Barrett's esophagus. Gastrointest Endosc. 2013;78:689-695.

4. Gupta, et al. Longer inspection time is associated with increased detection of high-grade dysplasia and esophageal adenocarcinoma in Barrett's esophagus. Gastrointest Endosc. 2012;76: 531-538.

5. Sharma $\mathrm{P}$, et al. The utility of a novel narrow band imaging endoscopy system in patients with Barrett's esophagus. Gastrointest Endosc. 2006;64:167-175.

6. Peters FP, et al. Surveillance history of endoscopically treated patients with early Barrett's neoplasia: nonadherence to the Seattle protocol leads to sampling error. Dis Esophagus. 2008; 21:475-479.

7. Rein BJ, et al. Optimizing endoscopic biopsy detection of early cancers in Barrett's high-grade dysplasia. Am J Gastroenterol. 2000;95:3089-3096.

8. Kandakandi V, Sharma P. Difficulties with a diagnosis of lowgrade dysplasia limit its utility as a risk stratification strategy in patients with Barrett's esophagus. https://www.infona.pl/ resource/bwmeta1 .element.elsevier-bb248013-2c64-3cba-899e-0 367b6c6aff3. https://www.infona.pl/resource/bwmeta1.element. elsevier-bb248013-2c64-3cba-899e-0367b6c6aff3.

9. Abdalla M, et al. Dysplasia detection rate of confirmatory EGD in nondysplastic Barrett's esophagus. Dis Esophagus. 2014;27: 505-510.

10. Abrams JA, et al. Adherence to biopsy guidelines for Barrett's esophagus surveillance in the community setting in the United States. Clinical Gastroenterol Hepatol. 2009;7:736-742.

11. Curvers, et al. Quality of Barrett's surveillance in the Netherlands: a standardized review of endoscopy and pathology reports. Eur J Gastroenterol Hepatol. 2008;20:601-607. 